\title{
Alternatives to surgery for patients with stridor secondary to multinodular goitres?
}

\author{
Maryam Adil, Georgina Page and Tristan Richardson \\ Bournemouth Diabetes and Endocrine Centre
}

The Royal Bournemouth Hospital

www.b-dec.co.uk

\section{Introduction}

Diffusely enlarged thyroid glands can cause compressive symptoms involving the trachea, oesophagus and recurrent laryngeal nerve. These symptoms range from mild, presenting with neck pressure or globus sensation, to severe, characterized by significant dysphagia or dyspnoea. ${ }^{1}$

We present a case of longstanding retrosternal goitre with compressive symptoms of dysphagia and dyspnoea.

\section{Case History}

Mrs DJ, an 88 year old lady, presented with gradually worsening stridor and dysphagia. Her past medical history was complex and included ischaemic heart disease and atrial fibrillation treated with warfarin.

She had been initially referred for enlargement of her longstanding goitre 6 years ago (2012) with investigations demonstrating a suppressed TSH and a normal FNA cytology. Respiratory function tests did not show any significant extra thoracic compression, but her CT scan confirmed a large goitre with retrosternal extension, tracheal deviation and narrowing (Fig. 1A\&B).

Although surgery was considered, in view of her multiple medical comorbidities, she underwent radioiodine treatment as she had been relatively asymptomatic. There was limited improvement in the goitre size but her thyroid function normalised.
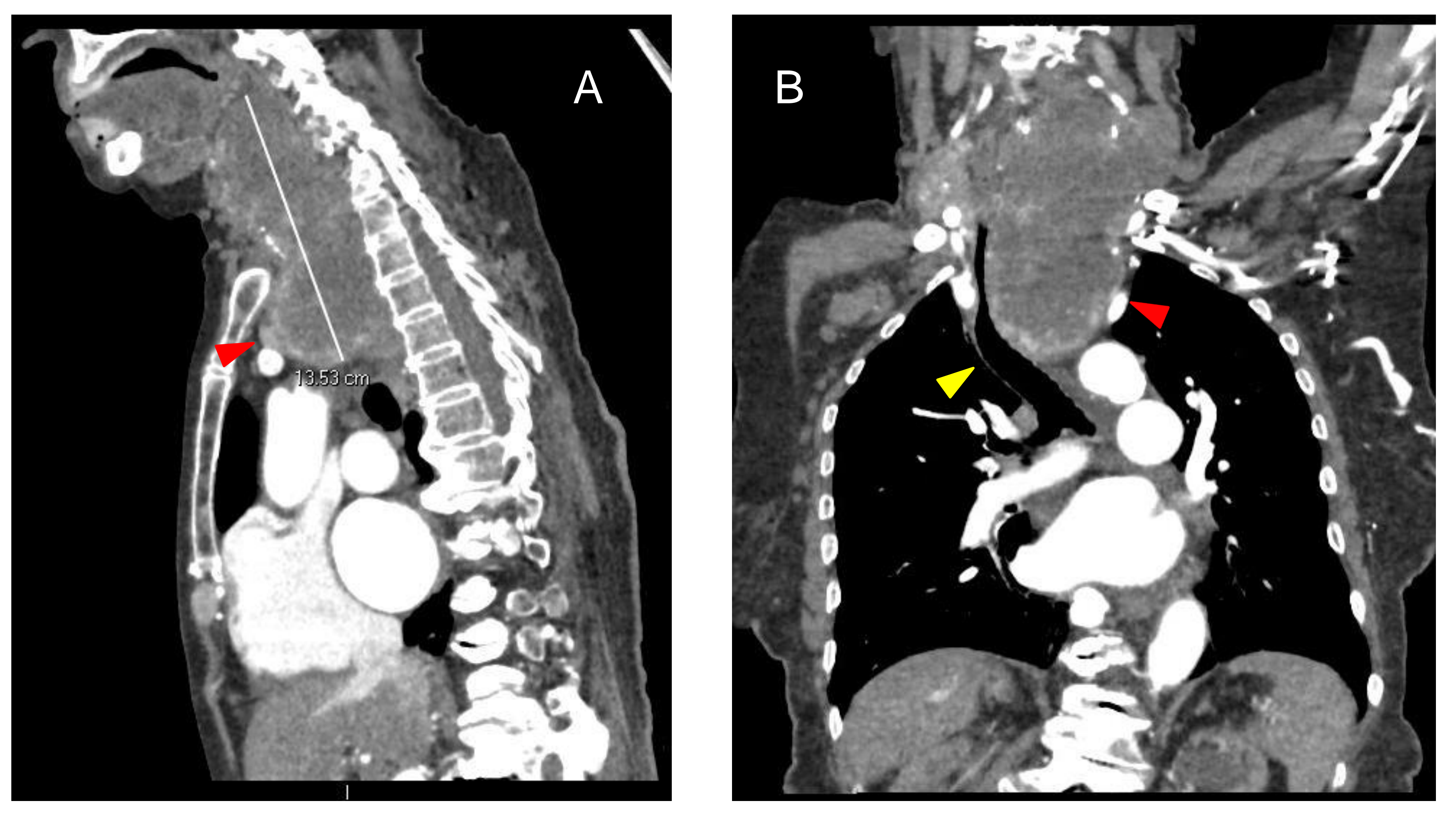

Fig. 1- CT chest, Sagittal (A) and Coronal (B), demonstrating retrosternal/intrathoracic extension of multinodular thyroid gland (Red arrowheads) with compression and displacement of the intrathoracic trachea to the right of midline. (Yellow arrowhead)

In 2017, she re-presented with a gradual increase in the size of her goitre associated with new onset stridor and dysphagia. In view of her comorbidities, anti coagulation, and the risks of surgery, she had tracheal stenting which successfully improved her symptoms.

\section{Discussion}

Surgery is considered as the mainstay treatment for patients with compressive symptoms secondary to retrosternal/mediastinal extension of thyroid gland, ${ }^{2}$ which not only requires high level of expertise but may also lead to significant complications. In elderly patients with multiple comorbidities, tracheal stenting could be considered as a useful alternative to surgery or radio-iodine.

\section{References}

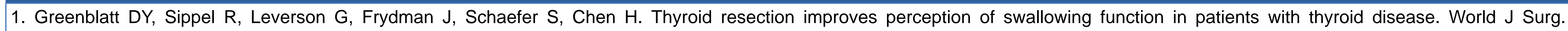
2009;33:255-60.

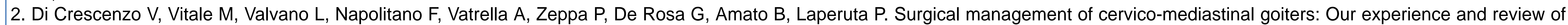
the literature. Int J Surg. 2016;28 Suppl1:S47-53. 